\title{
Análisis de Accesibilidad como Herramienta para la Generación de Zonas Libres de Tráfico. Caso del Municipio de La Dorada en Colombia
}

\author{
Santiago Cardona(1), Diego A. Escobar ${ }^{(1)}$ y Carlos A. Moncada(2) \\ (1) Universidad Nacional de Colombia, Sede Manizales. Fac. de Ingeniería y Arquitectura. Depto de \\ Ingeniería Civil. Cra 27 \# 64-60, Manizales, 170004, Colombia (email: scardonau@unal.edu.co; \\ daescobarga@unal.edu.co) \\ (2) Universidad Nacional de Colombia, Sede Bogotá. Fac. de Ingeniería. Depto de Ingeniería Civil y \\ Agrícola. Ciudad Universitaria, edificio 214, Oficina 417, Bogotá, 111321, Colombia. \\ (e-mail: camoncadaa@unal.edu.co)
}

Recibido Ene. 2, 2018; Aceptado Mar. 23, 2018; Versión final Jun. 8, 2018, Publicado Oct. 2018

\begin{abstract}
Resumen
Se realiza un análisis del impacto que puede generar los cambios de dirección y velocidad en la red vial del municipio de La Dorada en el departamento de Caldas (Colombia). Para ello se emplean modelos de oferta del transporte, a fin de generar una zona libre de flujo vehicular en el área del Malecón donde puedan potenciarse los espacios públicos y de recreación para los ciudadanos. Mediante un análisis de accesibilidad para cuatro escenarios, la situación actual y tres alternativas de intervención, se calcula el porcentaje de ahorro en el tiempo medio de viaje en la situación base y en cada una de las alternativas. Los resultados obtenidos demuestran que una correcta combinación de los cambios de dirección y cambios de velocidad en la red vial puede liberar el flujo de vehículos de una zona de la ciudad debido al ahorro de tiempo al utilizar rutas diferentes para desplazarse.
\end{abstract}

Palabras clave: accesibilidad; geoestadística; sentido vial; planeamiento municipal; transporte

\section{Accessibility analysis as a tool for the generation of traffic free zones. Case study: La Dorada, Colombia}

\begin{abstract}
.
An analysis of the impact that can generate changes of speed and of transit direction in the road network of the municipality of La Dorada in the department of Caldas (Colombia) is presented and discussed. This is done employing models of transport, in order to generate a free-zone of vehicular flow in the Malecón area where public spaces and recreation for citizens can be enhanced. Through an accessibility analysis in four scenarios, the current situation and three intervention alternatives, the percentage of savings in the average travel time in the base situation and in every alternative scenario is calculated. The results show that a correct combination of direction changes and speed changes in the road network can decrease the flow of vehicles of a city area due to the saving of time when using other different routes to move.
\end{abstract}

Keywords: accessibility; geo-statistics; road direction; local planning; transport. 


\section{INTRODUCCIÓN}

El municipio de La Dorada, se localiza en el Departamento de Caldas a $5^{\circ} 27^{\prime \prime}$ latitud norte y $74^{\circ} 40^{\prime \prime}$ de longitud este del meridiano de Greenwich en la zona centro de Colombia (Ver Figura 1). La Dorada tiene una extensión de $574 \mathrm{~km} 2$ y se ubica a una altura de $178 \mathrm{msnm}$ sobre la margen izquierda del río Magdalena, el más importante a nivel fluvial del país. Este municipio posee una ubicación estratégica que le ha brindado el calificativo de "Glorieta Nacional" o "Corazón de Colombia" lo que le permite articularse perfectamente al desarrollo de regiones al oriente de Caldas, norte Tolima, suroccidente de Santander, noroccidente de Cundinamarca, suroriente de Antioquia y occidente de Boyacá y Cundinamarca (La Dorada, 2013).

En el municipio de La Dorada una parte de su infraestructura está pensada como vía de margen paisajístico mediante intervenciones a la infraestructura vial y el espacio público que pretenden ser un potenciador ambiental, comercial y paisajístico de la zona, con el fin de convertirle en un verdadero atractivo turístico y recreativo que sea frecuentado por un importante número de personas, dado lo anterior, el disminuir el flujo vehicular que atraviesa este sector y disminuir la velocidad promedio de los vehículos automotores, es de vital importancia para aumentar la seguridad vial y hacer más atractivo el lugar. En la figura 2 se puede observar la zona de estudio del Malecón ubicada en la parte sur del municipio y que conecta los barrios de esta parte de la ciudad con el centro del mismo; actualmente esta vía refiere congestión, y dada la vocación comercial y de servicios del sector, es necesario que exista por lo menos un carril para vehículos livianos que se desplacen a baja velocidad, así como algunas bahías para los proceso de carga y descarga de mercancías en el sector, tratándose de ganar más espacio para el disfrute del peatón.

Por lo tanto, en este estudio se pretende realizar un análisis de re direccionamiento e intervención en la infraestructura vial con la finalidad de generar que está zona tenga una liberación en el parque automotor que la frecuenta como alternativa de movilidad y así poder ampliar el espacio público urbano disponible para peatones y vehículos no-motorizados como bicicletas. La intervención en la infraestructura vial será analizada desde una perspectiva futura donde la zona del Malecón será semi peatonalizada disminuyendo su velocidad promedio y reemplazando su capa de rodadura actual por adoquines. Además, se realiza un re direccionamiento convirtiendo esta vía en unidireccional de baja velocidad junto con cambios en algunas vías en la zona de influencia. El estudio se realizará mediante el análisis de accesibilidad media global, donde se evalúan los tiempos promedio de viaje en la red vial, entre la situación actual y diferentes alternativas de intervención donde se pueden registrar los impactos que generan las alternativas en la infraestructura operativa o física de la red vial (Escobar et al., 2015).
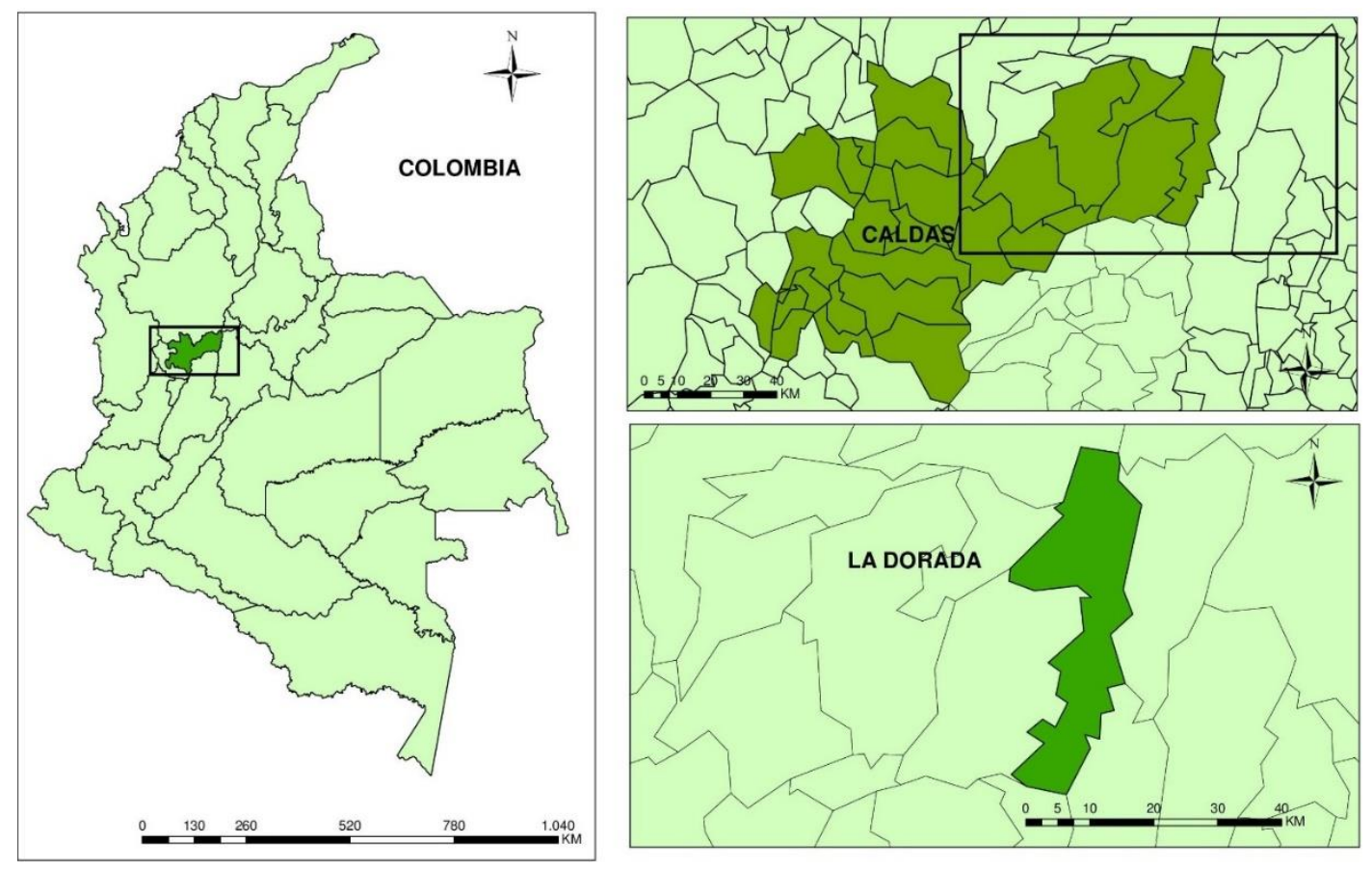

Fig.1. Ubicación geográfica del municipio La Dorada, Departamento Caldas, Colombia. Fuente: Elaboración Propia.

La pregunta concreta de investigación radica en que a partir de la aplicación de metodologías de accesibilidad territorial se puede establecer el impacto que generaría un redireccionamiento vial en la ciudad y por lo tanto la generación de zonas libres de tráfico vehicular. Luego de la breve introducción se presenta un apartado del 
estado de la cuestión, la metodología de investigación, posteriormente se muestran los resultados obtenidos y discusión de éstos, para finalmente abordar las principales conclusiones del estudio.

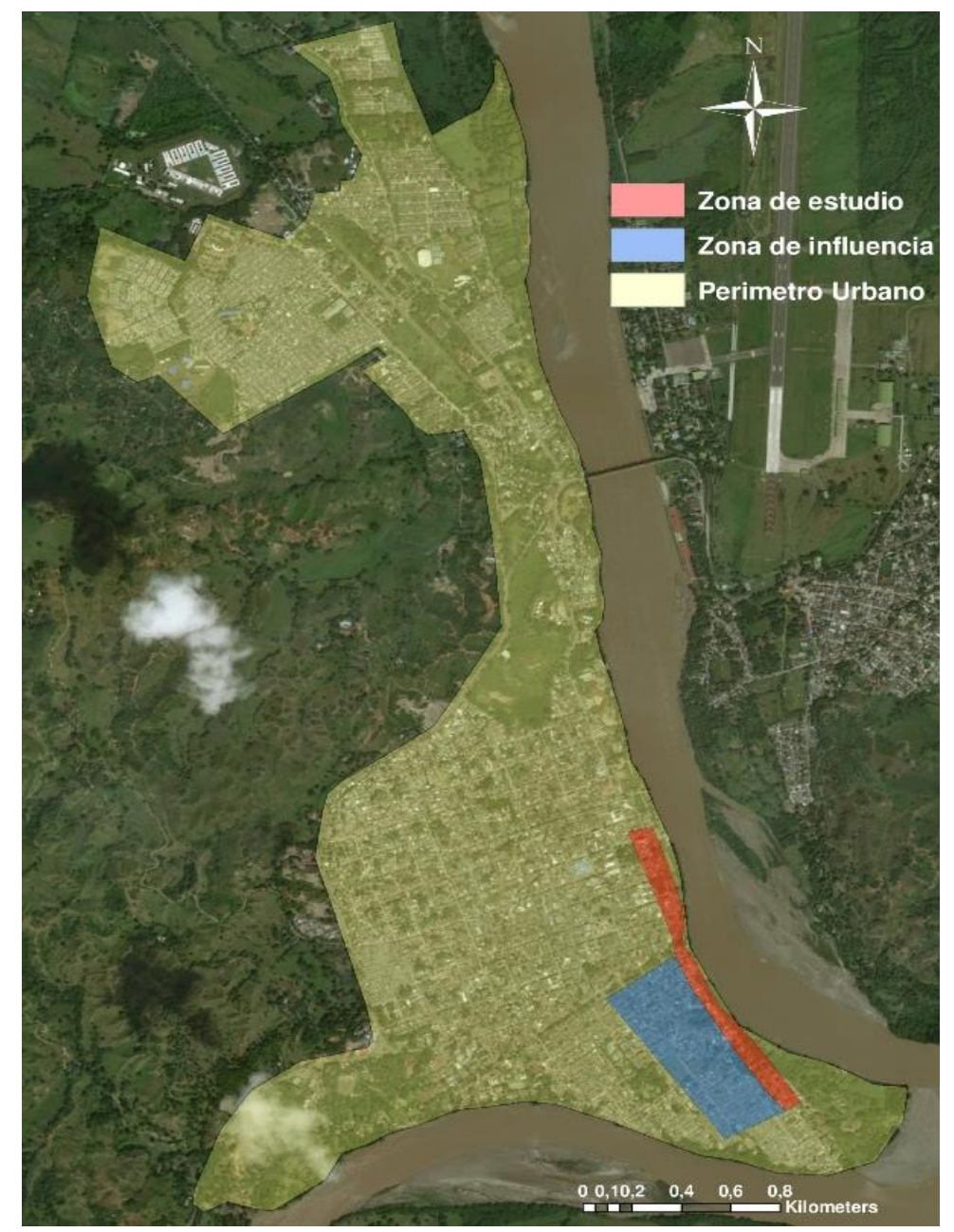

Fig.2. Zona de estudio y área de influencia. Fuente: Elaboración propia.

\section{ESTADO DE LA CUESTIÓN}

La accesibilidad es un concepto que surgió durante la segunda década del siglo pasado (Batty, 2009), no obstante, se considera que la definición más clásica del concepto es “...el potencial de oportunidades para la interacción..." expresada por Hansen (1959). El potencial de oportunidades se puede observar desde distintos puntos de vista que contribuyen al análisis como los propósitos y motivación del viaje, los modos de viaje, tipos de viajeros, edad, género, grupos ocupacionales, etc. (Pirie, 1979). Así, se puede realizar un estudio de accesibilidad territorial urbana donde se tenga en cuenta las mujeres que utilizan el transporte público para ir al trabajo diferente a las que utilizan el vehículo privado o la bicicleta como modo de viaje, por ejemplo.

En el ámbito de la literatura las medidas de accesibilidad se pueden dividir en tres tipos principales: accesibilidad relativa, accesibilidad integral y accesibilidad global. La accesibilidad relativa se refiere a la medida entre dos puntos A y B particulares de la red de infraestructura en una ciudad, por lo tanto, la medida de accesibilidad desde A hasta B será igual que la medida de accesibilidad tomada desde B hasta A (Ingram, 1971). La accesibilidad integral es aquella medida que se toma desde un punto de la red que representa los habitantes $o$ un grupo de ellos respecto a un conjunto de puntos que representan las oportunidades tales como la educación, trabajos, salud, recreación, entre otros. (Ingram, 1971, Handy y Niemeier, 1997). Está medida posee gran popularidad y por este motivo se han desarrollado diferentes modelos como el basado en oportunidades que básicamente distingue el número de oportunidades potenciales que existe en el área de estudio, el modelo basado en gravedad, además de tener en cuenta el número de oportunidades, también hace uso de la variable distancia y tiempo de viaje y por último el modelo basado en la utilidad que incluye variables de deseo donde las personas perciben mayor atractividad de ciertos lugares debido a distintos factores como la distancia, el tiempo, el precio, la oferta de servicios, entre otros. Este método es muy popular para determinar variables económicas en las ciudades y sus potenciales oportunidades (Hansen, 1959; Pirie, 1979; Handy y Niemeier, 1997, Geurs y Ritsema van Eck, 2001). La accesibilidad media global resulta de 
hacer una medición en el tiempo de viaje entre todos los nodos de la red vial, siendo de gran utilidad para analizar el comportamiento que presentan nuevas obras de infraestructura o cambio de direccionalidad en la misma (Escobar y García, 2012). Es claro precisar que las medidas de accesibilidad toman gran importancia en la planificación del territorio y como insumo fundamental para la toma de decisiones de los planificadores de la ciudad ya qué permiten identificar las áreas del territorio y segmentos de la población que poseen falencias de acceso a los bienes y servicios de la ciudad (Guzmán et al., 2017) o el impacto que generarán nuevas obras de infraestructura en la red vial de la ciudad (Escobar y García, 2012).

Por otra parte, en la literatura se encuentran diferentes estudios que relacionan la accesibilidad con temas como el desarrollo del suelo y la planificación de ciudad (Geurs y Van Wee; 2004), desarrollo sustentable del transporte (Kwok y Yeh; 2004; Cheng et al., 2007, Vega, 2011), exclusión social (Preston y Rajé, 2007), planificación de transporte público (Gulhan et al., 2013), acceso a sistemas de salud (Escobar et al., 2016), estudios de redireccionamiento vial (Escobar et. al., 2014; Barco et. al., 2018), desarrollo económico (Holl, 2007; MacKinnon et al., 2008; Ribeiro y Silva, 2011), cohesión social (Schürman et al.,1997; López et al., 2008; Farrington y Farrington, 2005), prestación y localización de servicios (Calcuttawala, 2006; Park, 2012; Higgs et. al., 2013), entre muchos otros estudios.

En relación con estudios que aborden el tema de análisis de accesibilidad y su influencia como variable definidora de zonas libres de tráfico, la literatura es poca, lo cual refuerza que la presente investigación se propone como una metodología novedosa que puede apoyar en algún momento la toma de decisiones por parte de la administración municipal; no obstante, se han encontrado algunas investigaciones que analizan zonas de congestionamiento realizando propuestas para liberar áreas específicas del efecto directo de los vehículos automotores (Asaithambia y Basheerb, 2017), e investigaciones que proponen sistemas de redireccionamiento de tráfico que aunque no se soportan en análisis de accesibilidad, presentan nuevas metodologías (Bazan et al., 2017); así mismo, se debe destacar que los análisis de accesibilidad igualmente han sido un soporte técnico importante al momento de estudiar el impacto de las modificaciones realizadas a la red vial (Hartmanna y Yeang Yng Lingb, 2016), los cual influye en la posible generación o no de este tipo de zonas libres de tráfico.

\section{METODOLOGIA}

A continuación se describe la metodología propuesta para la investigación, la cual consta de seis (6) etapas consecutivas que se muestran en la figura 3.

Etapa 1, Definición de la zona de estudio y toma de datos en campo. Se realizó toma de datos en campo de velocidades, lo cual consistió en el registro del tiempo que le tomaba a los vehículos atravesar una distancia conocida, en diez (10) arcos de la red vial para luego realizar el cálculo de la velocidad media, insumo fundamental para el cálculo de la accesibilidad ya que permite obtener el tiempo de viaje para cada arco en la red. Por otro lado, se registró el inventario de infraestructura vial para conocer el estado actual de la malla vial, las señales de tránsito y los andenes.

Etapa 2, Georeferenciación de la red vial y cálculo de matriz de tiempos. Para la creación de la red vial del municipio se usó un Sistema de información geográfica (SIG) que permitió obtener una red georreferenciada con aproximadamente 1570 arcos y 964 nodos, verificada gracias a diferentes mapas obtenidos del Plan Básico de Ordenamiento Territorial (PBOT) 2013-2017 del municipio de la Dorada y herramientas de acceso libre en internet como Google Maps y OpenStreetMaps. Para el cálculo de la matriz de tiempos de viaje es necesario la obtención del tiempo viaje de cada arco el cual es calculado gracias a la longitud de los arcos y a las velocidades tomadas en campo y que fueron replicadas en los demás arcos de la red según el mapa de jerarquización vial del municipio. La vía que recorre el sector de estudio está categorizada como una vía marginal paisajística, categorización que difiere con la realidad encontrada en ésta, alta velocidad y alto flujo de vehículos, encontrando que la razón por la cual la administración municipal asignó dicha categoría, es por la capacidad de atracción peatonal del sector, así como la oferta de espacio público y servicios recreacionales. Luego de obtener el tiempo de viaje de cada arco se puede calcular la matriz de tiempos de viaje (tvi), es decir, lo que le tomaría a cada nodo llegar a cada uno de los nodos restantes de la red mediante el algoritmo de Dkjistra el cual es el encargado de calcular la ruta minimizando el tiempo de viaje.

Etapa 3, cálculo de la accesibilidad media global. Para el cálculo de la accesibilidad media global es necesario obtener el tiempo promedio de viaje de cada nodo (Tvi, Expresión 1), a partir de la matriz calculada en la etapa 2. El vector se relaciona con las coordenadas geográficas de los mismos con el fin de obtener las curvas isócronas de accesibilidad media global mediante la aplicación del modelo geoestadístico (Zuluaga y Escobar, 2016). 


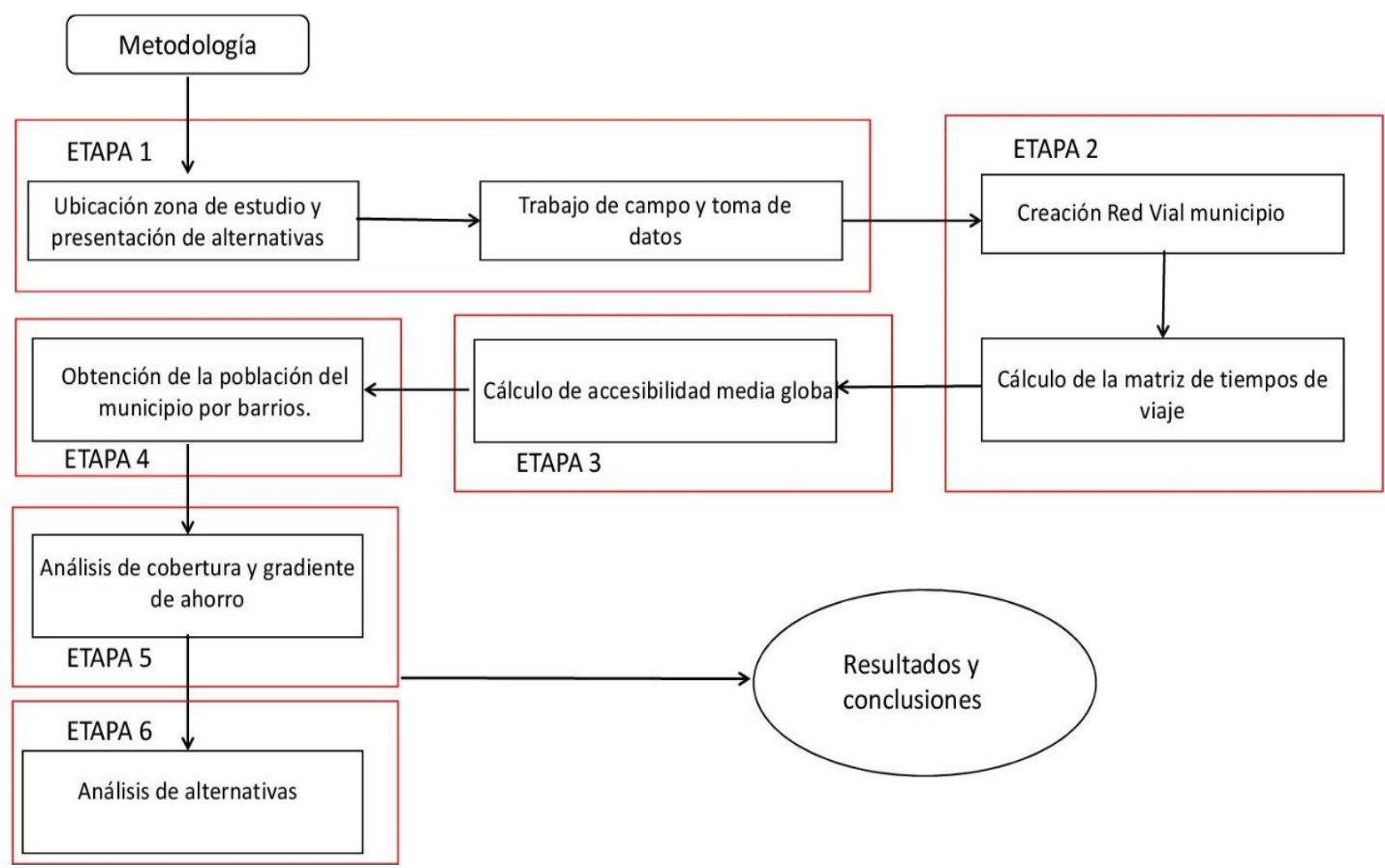

Fig. 3. Metodología abordada en el estudio. Fuente: Elaboración Propia.

$\overline{T v i}=\frac{\sum_{j=1}^{n} t v i}{n-1} \quad i=1,2,3 \ldots, n ; j=1,2,3, \ldots, n$

Para los cálculos geoestadísticos de predicción se optó por utilizar el Método Kriging ordinario el cual propone una predicción mediante la combinación lineal de las $n$ variables aleatorias $(T V I)\left(Z^{*}(x 0)\right.$, Expresión 2) donde $\lambda_{i}$ representa las ponderaciones de los valores originales y es calculada en función de la distancia entre los nodos y los puntos donde se pretende hacer la predicción. La suma de estás ponderaciones debe ser igual a la esperanza variable $\left(E\left(T v_{o}\right)\right.$ ), la cual por el principio de insesgamiento es igual a uno (1) (Expresión 3). Además, como función de correlación espacial se utiliza el semivariograma $\left(\overline{\gamma_{(h)}}\right)$ el cual utiliza una función lineal para sus cálculos y comprende el criterio que a menor distancia entre los nodos mayor correlación espacial entre las observaciones (Expresión 4), lo que nos sugiere que para obtener mejores resultados en nuestra modelación la distancia entre nodos debe ser la menor posible (Giraldo, 2002; Wackernagel, 2003).

$$
\begin{aligned}
& T_{0}=\lambda_{1} T v_{1}+\lambda_{2} T v_{2}+\lambda_{3} T v_{3}+\lambda_{4} T v_{4}+. .+\lambda_{n} T v_{n}=\sum_{i=1}^{n} \lambda_{i} T v_{i} \\
& E\left(T v_{0}^{*}\right)=E\left(T v_{o}\right) \\
& \overline{\gamma_{(h)}}=\frac{\sum\left(Z_{(x+h)}-Z_{(x)}\right)^{2}}{2 n}
\end{aligned}
$$

Etapa 4, información demográfica. La obtención de la población del municipio por barrios (Ver figura 4) comprendió distintas fases. En la primera de ellas se obtuvieron la extensión, forma y nombre de los barrios gracias al PBOT. En la segunda se obtuvo un raster de precisión a 100×100 metros desde la página WorldPop, la cual posee datos abiertos como la densidad, tasa de natalidad, defunciones, enfermedades, etc. en diferentes países del mundo. En el caso de Colombia se pudo obtener la densidad poblacional la cual fue comparada con el área de los barrios para así calcular la cantidad de habitantes en cada uno de ellos. Cabe resaltar que el resultado final fue de 84 mil habitantes, dato cercano al registrado por el DANE de 85677 habitantes en el perímetro urbano del municipio para 2015. Todo lo anterior con el fin de tener la población aproximada por barrio, lo cual es información que será usada para los cálculos de cobertura en relación con las curvas isócronas obtenidas para cada alternativa estudiada. 


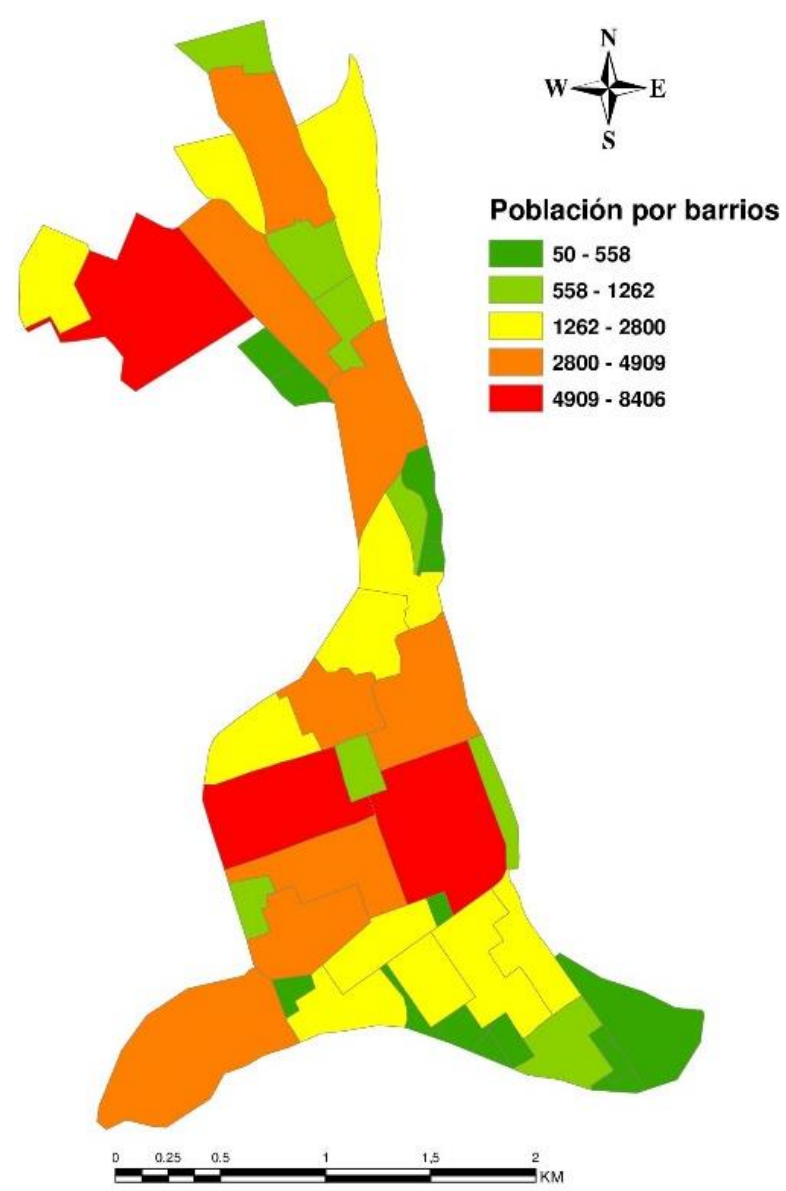

Fig. 4. Población del municipio por barrios. Fuente: Elaboración Propia.

Etapa 5, análisis de cobertura y gradiente de ahorro. Para la obtención del gradiente de ahorro (\%Gah) se realiza un cálculo entre los resultados de la accesibilidad media global para la situación base ( $A M G b)$ y cada una de las alternativas propuestas $(A M G x)$ (Expresión 5). Así, se obtiene un mapa por cada alternativa donde se puede evidenciar el porcentaje de ahorro de tiempo promedio de viaje que se tendrían dadas las intervenciones de redireccionamiento propuestas en la infraestructura vial.

$$
\% G a h=\left(\frac{A M G b-A M G x}{A M G b}\right) * 100
$$

Etapa 6, comparación de alternativas. En la comparación de alternativas se tiene en cuenta el gradiente de ahorro generado por cada una de éstas en la cobertura de población acumulada, es decir, tendría mayor valor la alternativa que refiera un menor tiempo medio de viaje cubriendo a un mayor porcentaje de población.

\section{RESULTADOS}

Mediante el análisis de velocidad de operación se pudo comprobar que en la zona del Malecón se registraron las velocidades promedio más altas de la red, lo que convierte a esta vía en la principal opción de ruta para las personas de la zona residencial ya que perciben un ahorro en su tiempo de viaje, dadas las características de la vía. A partir del trabajo de campo realizado se obtuvo que la categoría de vía arteria principal tiene una velocidad promedio de $43 \mathrm{Km} / \mathrm{h}$ (1185 velocidades de muestra con desviación estándar de 9,5 Km/h), vía colectora tiene una velocidad promedio de $34 \mathrm{Km} / \mathrm{h}$ (856 velocidades de muestra con desviación estándar de $7,9 \mathrm{Km} / \mathrm{h}$ ), vía local tiene una velocidad promedio de $24 \mathrm{Km} / \mathrm{h}$ (684 velocidades de muestra con desviación estándar de $8,2 \mathrm{Km} / \mathrm{h}$ ), y vía marginal paisajística tiene una velocidad promedio de $41 \mathrm{Km} / \mathrm{h}$ (526 velocidades de muestra con desviación estándar de $7,5 \mathrm{Km} / \mathrm{h}$ ); éste último tipo de vía es la que atraviesa la zona de estudio.

En la figura 5 se presenta la categorización y el direccionamiento actual de la red vial del municipio, la cual es base para realizar los cálculos de accesibilidad media global y la comparación del escenario actual con las tres alternativas de redireccionamiento que permitirán generar una zona libre de tráfico. Las tres alternativas analizadas se basan en a) redireccionamiento vial en la zona de estudio y área de influencia, b) aumento de 
velocidades promedio en los arcos de la red y c) una combinación de ambas. Se desataca que la velocidad promedio en el sector del Malecón fue disminuida a $20 \mathrm{~km} / \mathrm{h}$ y su dirección seria única en el sentido sur-norte en las tres (3) alternativas con el fin de modelar el escenario futuro de una futura semipeatonalización donde los vehículos perciban un importante aumento en los tiempos de viaje al utilizar está vía por lo que les lleve a decidir el uso de una ruta diferente liberando así de flujo vehicular este sector.

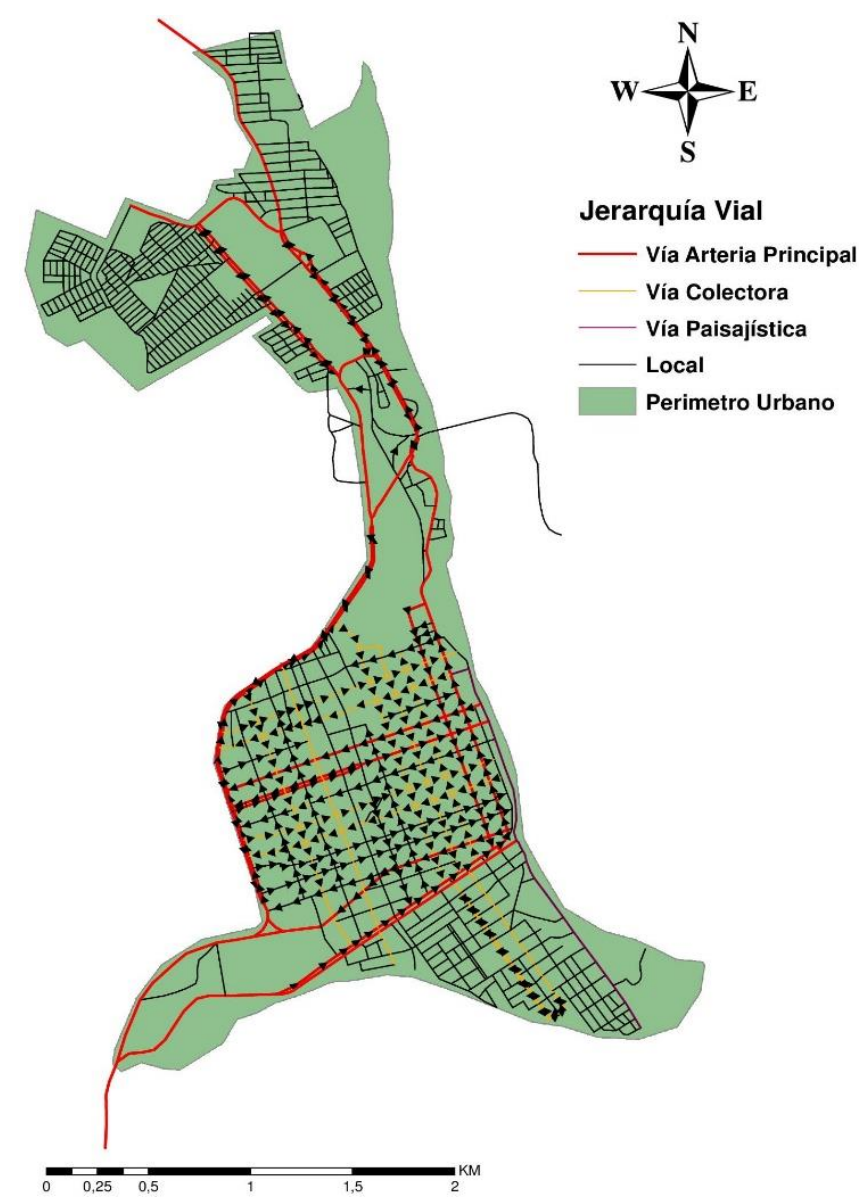

Fig. 5. Categorización y direccionamiento de la red vial del municipio. Fuente: Elaboración Propia.

En la figura 6 se observan las curvas isócronas de la accesibilidad media global para la situación base en el municipio, donde existen $\overline{T v i}$ entre 6 y 14 minutos. Se registran tiempos medios de viaje de 9 minutos cerca al sector central del municipio (Central Business District - CBD) y a medida que se acerca a la zona sur los tiempos medios de viaje aumentan hasta alcanzar los 12 minutos. Además, se puede notar que la zona que posee mayor accesibilidad global coincide con una vía arteria principal, la cual posee una velocidad alta y atraviesa el municipio de norte a sur conectando los barrios ubicados en el norte con el CBD y viceversa.

En la figura 7(a) se observa el gradiente de ahorro generado por el redireccionamiento vial de la alternativa 1 , donde se tienen gradientes negativos de hasta el $23 \%$ y gradientes de ahorro de hasta el $4 \%$ en algunas zonas del municipio. El $70 \%$ de la población disminuye sus tiempos medios de viaje en valore da hasta el $4 \%$ en relación con el escenario actual, lo cual demuestra que los cambios en la direccionalidad pueden ser medidas que beneficien a un gran porcentaje de la población. En la figura 7 (b) se observa el gradiente de ahorro generado por los cambios de velocidad de la alternativa 2, donde se proponen mejorar las condiciones geométricas y de seguridad vial para así generar una velocidad promedio más alta en algunas vías arterias principales y colectoras del municipio.

En este caso, el $75 \%$ de la población se ve beneficiada por unos ahorros en los tiempos medios de viaje, identificando ésta como una de las alternativas aceptables para la liberación de flujo en la zona del Malecón debido a que allí se ven aumentados los tiempos lo que ocasiona que el volumen de tráfico se traslade a lugares o rutas donde el tiempo de viaje es igual o mejor. Por otro lado, tan solo el $13 \%$ de la población sufre perdidas en los tiempos promedio de viaje, alcanzando hasta un $16,9 \%$ de perdida, no obstante el grueso de la población que se ve afectada se encuentra en el intervalo de hasta el $2 \%$ de perdidas con un $11,5 \%$ de población. En la figura 7(c) se observa el gradiente de ahorro generado por los cambios de velocidad y direccionalidad propuestos en la alternativa 3 , en la cual se propone realizar una combinación de las dos alternativas anteriormente expuestas. 


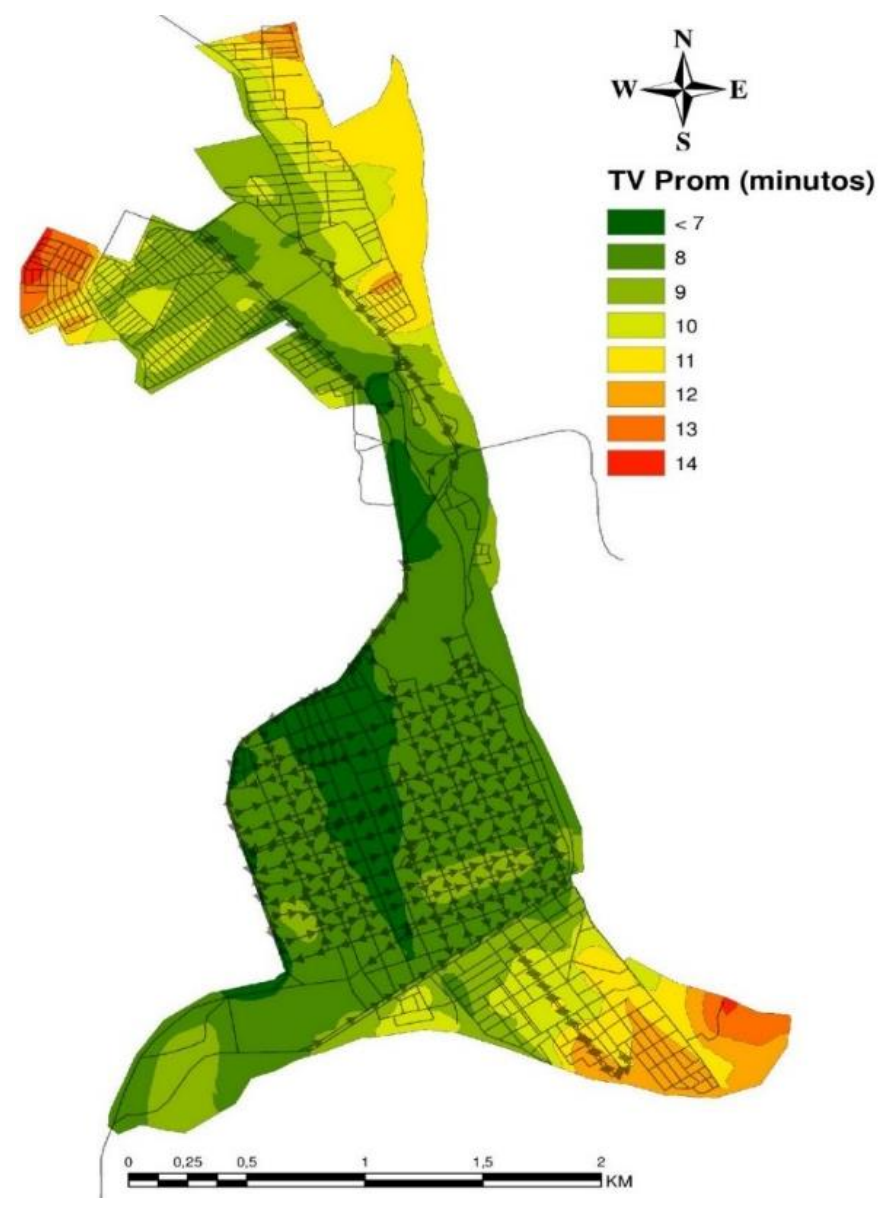

Fig. 6. Curvas isócronas de accesibilidad media global para la situación base. Fuente: Elaboración Propia.

En esta tercera alternativa se tiene como resultado que el $66 \%$ de la población obtiene ahorros en los tiempos medios de viaje, concentrado principalmente en los intervalos de ahorro entre 0 y $2 \%$ y $2 \%$ y $4 \%$, lo que nos indica que la ganancia neta de tiempo es poca, no obstante, se confirma que las intervenciones relacionadas con cambio direccional se ven mitigadas por el aumento de las velocidades en los arcos del municipio. Sin embargo, hay una parte de la población que sufre aumentos en el tiempo medio de viaje hasta en un $20 \%$ aunque para este intervalo no representa un porcentaje considerable, siendo menor al $1 \%$.

Es posible realizar una comparación de los resultados de cobertura en cada alternativa, como se observa en la figura 8 , en donde la ojiva que posea mayor pendiente refiere una mayor cobertura en tiempos medios de viaje menores. La alternativa 1 , donde sólo se realizan cambios en la direccionalidad es la que menos beneficia a la población en relación con la variable cobertura, no obstante, posee mayores probabilidades de reorganizar el tráfico a través de la red vial categorizada según funcionalidad. Por otro parte, la situación base posee grandes similitudes a las alternativas 2 y 3 en cuanto a tiempos de viaje lo que ocasiona que sus ojivas se encuentren muy cerca entre sí siendo la alternativa 2 , donde solo se realizan cambios de velocidades la que ocasiona un mayor beneficio en la población y aunque en la alternativa 3 se tienen en cuenta los mismos aumentos de velocidad, también se realizan cambios en la direccionalidad, lo que puede generar que los tiempos de viaje crezcan en algunos sectores debido a la disminución en las alternativas de ruta. Se recomienda entonces escoger aquella alternativa que combine el ahorro en los tiempos de viaje promedio en la mayor parte de la población y que suma en planificación y organización de la infraestructura vial del municipio. La alternativa 3 suma estás dos características debido a que beneficia con ahorro a dos tercios de la población y se realizan cambios en la direccionalidad que aumentan la seguridad vial de las intersecciones y la organización en la red municipal.

\section{DISCUSIÓN FINAL}

Las tres alternativas de intervención estudiadas, pueden ser analizadas desde diferentes puntos de vista. La primera alternativa, en la cual se realizan modificaciones en la direccionalidad de las vías en la zona de influencia, resulta poco impactante en términos de ahorro en tiempo medio de viaje al compararla con las otras dos alternativas analizadas. Esto se debe a que en la zona de influencia se detectó, a partir del trabajo de campo (información primaria), que la mayor cantidad de arcos son bidireccionales, lo cual trae como 
consecuencia que su modificación a ser arcos unidireccionales supone no sólo una disminución en el número de alternativas para alcanzar dicha zona, sino también un aumento en los recorridos de los usuarios, lo cual depende desde qué sector de la ciudad se realicen los desplazamientos.

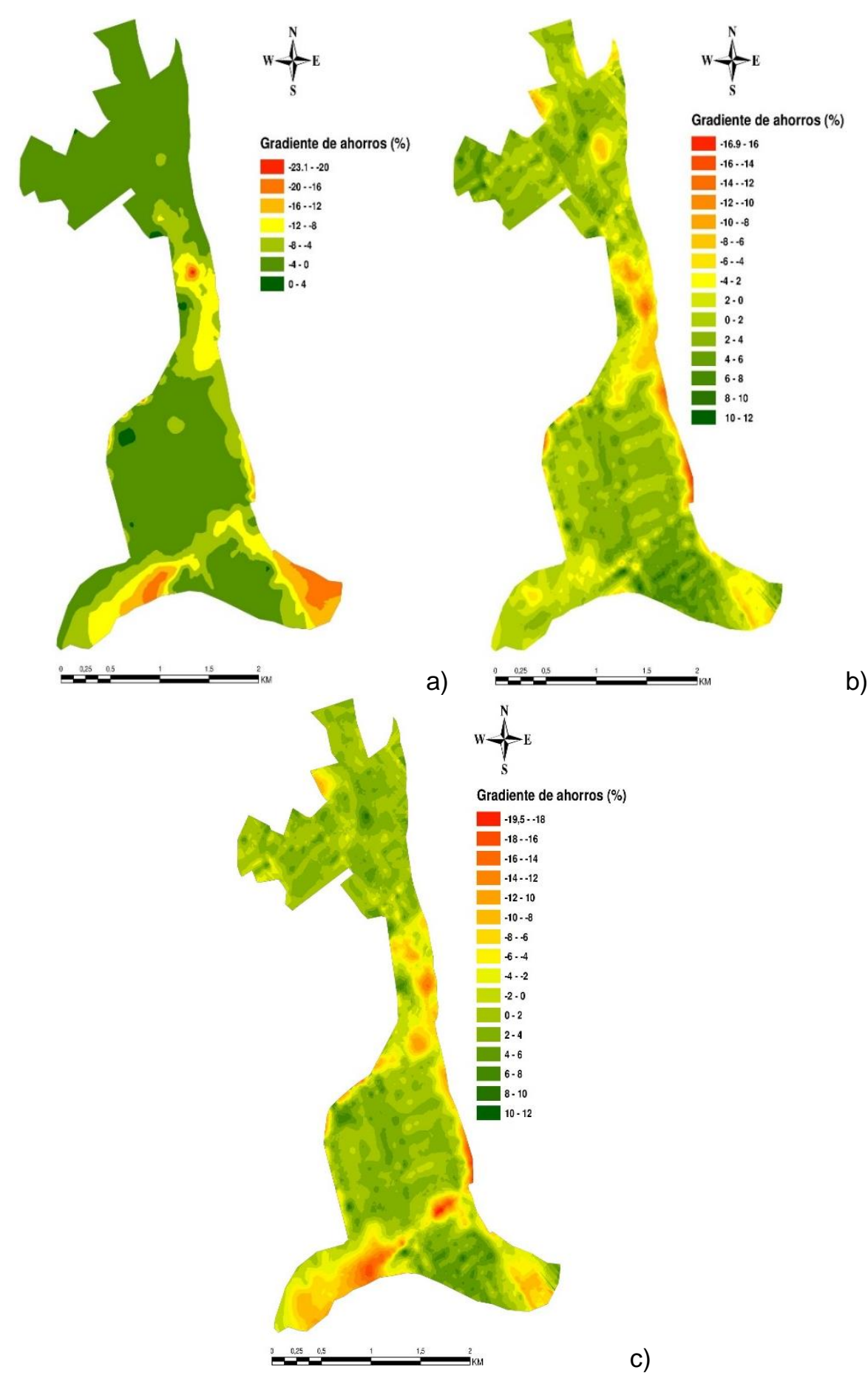

Fig. 7. Gradiente de ahorro generado por las tres alternativas respecto a la situación base. (a) Redireccionamiento vial; (b) cambios de velocidad; (c) redireccionamiento y cambios de velocidad. Fuente: Elaboración Propia.

La segunda alternativa, basada en modificar las características operativas de la red (aumento de velocidad) mediante el mejoramiento de las condiciones de la infraestructura vial, capa de rodadura, andenes y principalmente demarcación y señalización horizontal y vertical, arrojó como resultado un ahorro de tiempo medio de viaje para un gran porcentaje de la población; no obstante, se identifica una pérdida puntual de tiempo medio de viaje en el sector del Malecón debido a que en dicho sector de intervención se propone controlar y gestionar las características operativas de la red (disminución de velocidad). Está alternativa resulta beneficiosa en si misma a nivel de ciudad, a pesar de que el sector del malecón no se vea directamente beneficiado en la reducción del tiempo medio de viaje, lo cual, de forma indirecta ayudaría a que algún 
porcentaje de las personas que deseen pasear por el malecón lleguen al mismo en otros modos de transporte distintos al vehículo privado, situación que finalmente potenciará la reactivación del sector y su transformación en una zona libre de tráfico vehicular. La tercera alternativa es la suma de las dos anteriores, basada en cambios de características operativas de la red (modificaciones en velocidad) y en cambios de direccionalidad, encontrando que gran parte de la población refiere algún porcentaje de ahorro en el tiempo medio de viaje para alcanzar el sector de estudio.

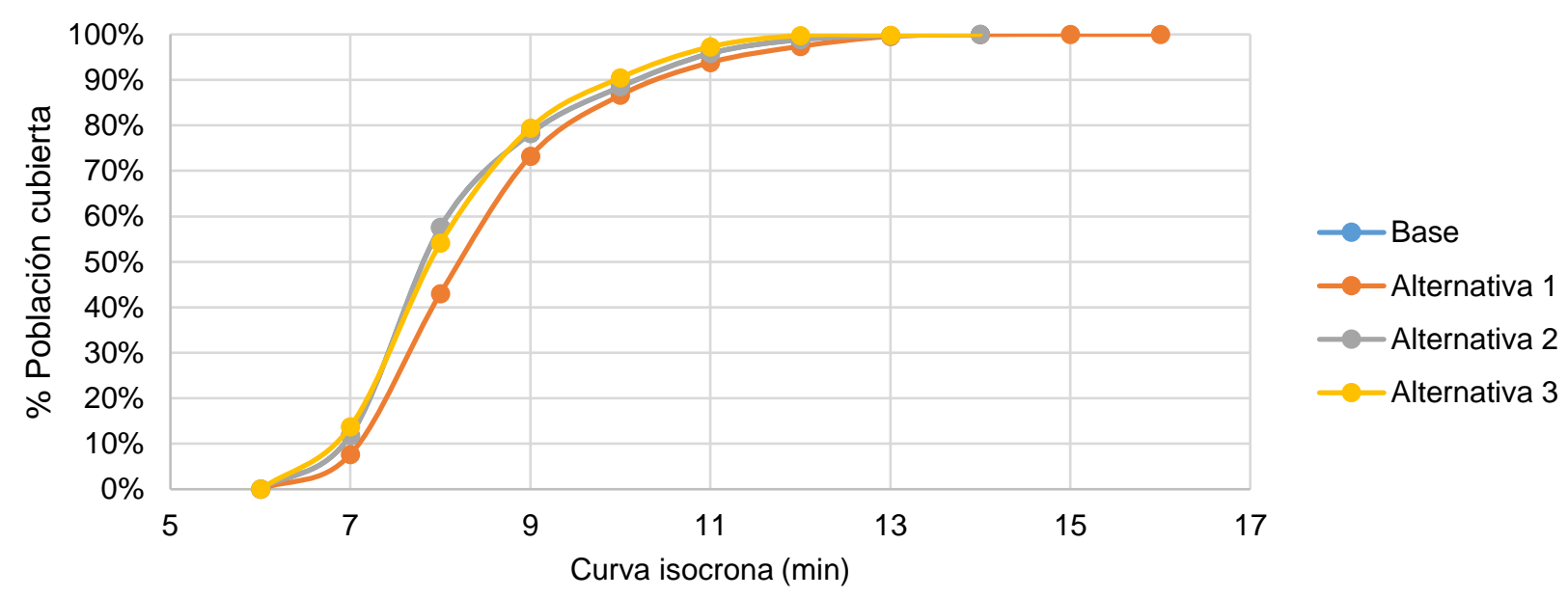

Fig.8. Ojiva porcentual de cobertura de población Vs curva isócrona (min). Fuente: Elaboración Propia.

Se destaca que el modificar los arcos de bidireccionales a unidireccionales genera aumentos en los tiempos promedio de viaje debido a que la versatilidad de la red de infraestructuras se ve disminuida al generar la necesidad de realizarse recorridos más largos para conectar algunos nodos en específico. No obstante, el establecer arcos unidireccionales, de forma indirecta disminuye las probabilidades de que se presente accidentes de tránsito al disminuirse el número de movimientos convergentes en las intersecciones. Para generar una zona libre de tráfico vehicular es indispensable sumar distintas acciones como la disminución de la velocidad de operación promedio mediante el cambio de superficie de rodadura, disminución del ancho de carriles y aumento del espacio público destinado al peatón y al ciclista además de generar alternativas de ruta para que los tiempos medios de viaje no se vean afectados en gran parte de la población, lo cual se logra al generar vías cuya función operativa sea la de alojar un flujo vehicular que se vea afectado por la disminución de sus características operativas, como es el caso de las vías circundantes al Malecón.

Durante la realización de la investigación no se tuvieron dificultades directamente relacionadas con la obtención de la información primaria (velocidad de operación vehicular, características físicas de la infraestructura, etc.); no obstante, es importante mencionar al tener la posibilidad de tener los datos de características operativas de la red de infraestructuras sólo para un porcentaje de la misma o para puntos o tramos específicos de esta, le convierte en una carencia metodológica que podría ser solventada mediante el uso de GPS o minería de datos de movilidad (inexistente para este ejemplo investigativo). Lo anterior obliga a que se deba asumir que las vías de igual categoría tengan hipotéticamente la misma velocidad de operación calculada para la vía escogida en el terreno. Por otra parte, valdría la pena establecer como una futura línea de investigación, el incluir factores externos de la infraestructura vial como número de carriles disponibles y número de carriles destinados para estacionamiento, con el fin de conocer su influencia sobre los resultados de accesibilidad.

\section{CONCLUSIONES}

De acuerdo al trabajo presentado y a los resultados obtenidos, se plantean las siguientes conclusiones principales:

1.- A partir análisis del estado del arte, fue posible establecer que son muy pocos los estudios que relacionan análisis de accesibilidad y definición de zonas libres de tráfico o estudios de redireccionamiento vial como tal, lo cual proporciona mucha más fuerza al artículo de investigación en relación de cómo al aplicar un instrumento de planificación urbana, como el análisis de accesibilidad territorial, se puede apoyar la toma de decisiones relacionadas con la definición de este tipo de zonas o sectores.

2.- Se concluye que la metodología aquí presentada y aplicada en la investigación se encuentra en su etapa preliminar, lo cual abre nuevas líneas de investigación futura que pueden complementar claramente el trabajo descrito; entre dichas líneas futuras de investigación estaría el efecto de proponer esta zonas libres de tráfico 
en la disminución de las emisiones de gases efecto invernadero, o su efecto directo sobre la influencia en los índices de accidentalidad vial, dado el aumento de la velocidad de operación en corredores viales definidos, así como el efecto que sobre las condiciones de estacionamiento se tendrían en el sector directamente definido como libre de tráfico y de cómo dicho parque automotor se reparte en el resto de la red vial.

3.- La aplicación de técnicas geoestadísticas en instrumentos de planificación territorial, como la aquí utilizada (Modelo Kriging), abre importantes posibilidades de análisis de diferentes alternativas de intervención sobre una red de transportes, donde a partir de interacciones sucesivas, las cuales pueden ser testadas en su aproximación, permitan definir cuál sería la mejor opción en el caso de buscarse algún tipo de efecto sobre un territorio. Con esta metodología se concluye sobre la posibilidad de aplicar de forma relativamente sencilla una herramienta que proporciona resultados bastante importantes en términos de análisis de ciudad. $S$ considera que la metodología puede ser aplicada con claridad en otras latitudes.

4.- Se concluye que los resultados obtenidos al aplicar la metodología descrita, aportan claridad sobre la pregunta de investigación en relación con la posibilidad de usar el procedimiento para evaluar diferentes opciones y configuraciones de red vial; es decir, la propuesta metodológica complementa los avances que sobre análisis de zonas o áreas de congestionamiento se tienen actualmente, pero observado desde el punto de vista de accesibilidad territorial.

\section{AGRADECIMIENTOS}

Los autores expresan su agradecimiento a los estudiantes pertenecientes a los semilleros de investigación en "Movilidad Sostenible" y "Planeación Urbana" de la Universidad Nacional de Colombia - Sede Manizales.

\section{REFERENCIAS}

Alcaldía de La Dorada, Información General (2013)

Asaithambia, G. y S. Basheerb, Analysis and Modeling of Vehicle Following Behavior in Mixed Traffic Conditions, https://doi.org/10.1016/j.trpro.2017.07.001, Transportation Research Procedia, 25, 5094-5103 (2017)

Batty, M., Accessibility: in search of a unified theory, doi:10.1068/b3602ed, Environment and Planning B: Planning and Design, 36(2), 191-194 (2009)

Barco, A., D. Escobar y C. Moncada, Road Redirection Analysis. Case Study: Araucarias Avenue, Manizales, Colombia, https://doi.org/10.12988/ces.2018.84154, Contemporary Engineering Sciences, 11(32), 1559-1569 (2018)

Bazan, M., T. Janiczek, K. Halawa, R. Dudek y L. Rudawski, Design and development of a road traffic redirection system, Archives of Transport Systems Telematics, 10(1), 3-8 (2017)

Calcuttawala, Z., Landscapes of information and consumption: A location analysis of public libraries in Calcutta, en Edward D. Garten, Delmus E. Williams, James M. Nyce (Eds.), Advances in Library Administration and Organization, Emerald Group Publishing Limited, 24, 319-388 (2006)

Cheng, J., L. Bertolini y F. le Clercq, Measuring sustainable accessibility, https://doi.org/10.3141/2017-03, Transportation Research Record: Journal of the Transportation Research Board, 2017, 16-25 (2007)

Escobar, D., J. Cañas y J.A. Montoya, Accesibilidad como herramienta de planeación urbana. Caso de estudio: Redireccionamiento vial en Riosucio (Caldas-Colombia), Avances investigación en ingeniería, 11(2) (2014)

Escobar, D. y F. García, Impacto regional: caso de aplicación de análisis de accesibilidad territorial, Revista Épsilon, 18 (2012)

Escobar, D., J. Holguin y J. Zuluaga, Accesibilidad de los centros de ambulancias y hospitales prestadores del servicio de urgencias y su relación con la inequidad espacial. Caso de estudio Manizales-Colombia, Revista Espacios, 37(20) (2016)

Farrington, J. y C. Farrington, Rural accessibility, social inclusion and social justice: towards conceptualization, doi: 10.1016/j.jtrangeo.2004.10.002, Journal of Transport Geography, 13(1), 1-12 (2005)

Geurs, K.T. y J.R. Ritsema van Eck, Accessibility Measures: Review and Applications. Evaluation of Accessibility Impacts of Land-use Transport Scenarios, and Related Social and Economic Impacts, National Institute of Public Health and the Environment, Utrecht, Netherland (2001)

Geurs, K.T. y B. Van Wee, Accessibility evaluation of land-use and transport strategies: review and research directions, doi: https://doi.org/10.1016/j.jtrangeo.2003.10.005, Journal of Transport Geography, 12(2), 127-140 (2004)

Giraldo, R., Introducción a la geo estadística: Teoría y aplicación, Universidad Nacional de Colombia, Colombia, Bogotá, Colombia (2002)

Gulhan, G., H. Ceylan, M. Özuysal, y H. Ceylan, Impact of utility-based accessibility measures on urban public transportation planning: A case study of Denizli, Turkey, http://dx.doi.org/10.1016/j.cities.2013.04.001, Cities, 32, 102-112 (2013)

Guzman, L., D. Oviedo y C. Rivera, Assessing equity in transport accessibility to work and study: The Bogotá region, https://doi.org/10.1016/j.jtrangeo.2016.12.016, Journal of Transport Geography, 58, 236-246 (2017) 
Handy, S. y D. Niemeier, Measuring accessibility: an exploration of issues and alternatives, https://doi.org/10.1068/a291175, Environment and planning A, 29(7), 1175-1194 (1997)

Hansen, W.G., How accessibility shapes land use, doi: https://doi.org/10.1080/01944365908978307, Journal of the American Institute of Planners, 25 (2), 73-76 (1959)

Hartmanna, A. y F. Yeang Yng Lingb, Value creation of road infrastructure networks: A structural equation approach, http://dx.doi.org/10.1016/j.jtte.2015.09.003, Journal of traffic and transportation engineering, 3(1), 28-36 (2016)

Higgs, G., M. Langford y R. Fry, Investigating variations in the provision of digital services in public libraries using networkbased GIS models, http://dx.doi.org/10.1016/j.lisr.2012.09.002, Library \& Information Science Research, 35(1), 24-32 (2013)

Holl, A., Twenty years of accessibility improvements. The case of the Spanish motorway building programme. doi:10.1016/j.jtrangeo.2006.09.003, Journal of Transport Geography, 15, 286-297 (2007)

Ingram, D., The concept of accessibility: a search for an operational form, https://doi.org/10.1080/09595237100185131, Regional studies, 5(2), 101-107 (1971)

Kwok, R. y A. Yeh, The use of modal accessibility gap as an indicator for sustainable transport development, https://doi.org/10.1068/a3673, Environment and Planning A, 36(5), 921-936 (2004)

López, E., J. Gutierrez y G. Gómez, Measuring regional cohesion effects of large-scale transport infrastructure investment: an accessibility approach, https://doi.org/10.1080/09654310701814629, European Planning Studies, 16(2), 277-301 (2008)

MacKinnon, D., G. Pirie y M. Gather, Transport and economic development. In R. Knowles, J. Shaw, \& I. Docherty, Editors, Transport Geographies: Mobilities, Flows and Spaces. 10-28, Blackwell Publishers, Oxford (2008)

Park, S., Measuring public library accessibility: a case study using GIS, https://doi.org/10.1016/j.lisr.2011.07.007, Library \& Information Science Research, 34(1), 13-21 (2012)

Pirie, G. H., Measuring accessibility: a review and proposal. Environment and Planning A, 11(3), $299-312$ (1979)

Preston, J. y F. Rajé, Accessibility, mobility and transport-related social exclusion, doi:10.1016/j.jtrangeo.2006.05.002, Journal of Transport Geography, 15(3), 151-160 (2007)

Ribeiro, A. y J. Silva, Space, development and accessibility between Portugal and Spain: the last frontier, Revista Portuguesa de Estudos Regionais, 27, 7-14 (2011)

Schürmann, C., K. Spiekermann y M. Wegener, Accessibility indicator, SASI Deliverable D5, Report to the European Commission, Berichte aus dem Institut für Raumplanung, 39, Dortmund (1997)

Vega, A., A multi-modal approach to sustainable accessibility in Galway, https:// doi.org/10.1080/20429843.2011.9727923, Regional Insights, 2(2), 15-17 (2011)

Wackernagel, H. Multivariate geostatistics: an introduction with applications, Springer Science \& Business Media, Berlin, Alemania (2003)

Zuluaga, J. y D. Escobar, Análisis de accesibilidad territorial de la región noroccidente de Colombia. Revista Espacios, 38(06) (2017) 